\title{
Posterior reversible encephalopathy syndrome in una paziente con AIDS, insufficienza renale acuta, ipertensione arteriosa
}

\author{
Posterior reversible encephalopathy syndrome in an AIDS patient \\ with acute renal failure and hypertension
}

\section{Olivia Bargiacchi, Anna M. Salerno, Antonella Rossati, Roberta Brondolo, Diego Brustia, Felicita Rosa, Giovanni Rizzo, Pietro L. Garavelli*}

SC Malattie Infettive, AOU Maggiore della Carità, Novara

Ricevuto il 15 novembre 2010; accettato il 21 gennaio 2011

disponibile online il 25 febbraio 2011

\section{KEYWORDS}

Posterior leukoencephalopathy syndrome;

HIV.

\begin{abstract}
Summary
Introduction: The posterior reversible encephalopathy syndrome (PRES) is a neurological entity characterized by magnetic resonance imaging (MRI) evidence of bilateral subcortical edema in the occipital regions of the brain.

Case report: We report the case of a female patient with AIDS, pulmonary aspergillosis, CMV infection, and acute renal failure due to Clostridium difficile diarrhea. Her clinical course was complicated by seizures and hypertension. MRI findings were consistent with PRES. The patient was treated with anticonvulsants and antihypertensive agents with clinical improvement. Discussion and conclusions: Few cases of PRES in HIV-infected patients have been described, and it is not clear whether HIV infection is a predisposing factor for this syndrome. The article reviews the literature on PRES in HIV and discusses the role of HIV-associated endothelial damage in the pathogenesis of this syndrome.

(c) 2011 Elsevier Srl. All rights reserved.
\end{abstract}

\section{Introduzione}

La Posterior Reversible Encephalopathy Syndrome (PRES) è un quadro neurologico caratterizzato da ipertensione, nausea, vomito, disturbi visivi, stato confusionale, crisi epilettiche, letargia o coma. È stata associata a condizioni cliniche quali preeclampsia/eclampsia, trapianto allogenico di midollo osseo o d'organo solido, malattie autoimmuni,

\footnotetext{
* Corrispondenza: via Comunale 73C - 15122 Valmadonna (AL).

E-mail: pietro.luigi.garavelli@maggioreosp.novara.it (P.L. Garavelli).
} 
tossicità da chemioterapici che attraverso il danno endoteliale e l'edema vasogenico possono determinare l'insorgenza delle manifestazioni cliniche.

In letteratura sono riportati alcuni casi di PRES in pazienti HIV positivi e, sebbene non siano chiari i meccanismi fisiopatologici che possono scatenare questa sindrome, si pensa che nella sua patogenesi possano essere coinvolti le alterazioni metaboliche indotte dalla terapia antiretrovirale o il danno endoteliale indotto dallo stato di immunoattivazione nelle forme avanzate.

Descriviamo un caso clinico di PRES in una paziente sieropositiva per HIV, gravemente immunodepressa.

Gli autori dichiarano che lo studio presentato è stato realizzato in accordo con gli standard etici stabiliti nella Dichiarazione di Helsinki, e che il consenso informato è stato ottenuto da tutti i partecipanti prima del loro arruolamento allo studio.

\section{Caso clinico}

Una donna di 35 anni di origine africana giunge alla nostra attenzione nel 2003 per riscontro di sieropositività per HIV. Per circa 5 anni la paziente si presenta regolarmente alle visite programmate, ma con un buon compenso immunovirologico, per cui non viene iniziato alcun trattamento antiretrovirale. Dopo 2 anni in cui la paziente non esegue più controlli ambulatoriali, si ripresenta per una sindrome diarroica e viene ricoverata presso il reparto Malattie Infettive dell'Ospedale "Maggiore della Carità" di Novara.

Al momento del ricovero la paziente appare poco collaborante, ma vigile, orientata nello spazio e nel tempo. L'esame obiettivo evidenzia epatosplenomegalia e ipotensione. Gli esami ematochimici mostrano una marcata pancitopenia ed elevati valori di creatininemia determinati da un'insufficienza renale acuta, verosimilmente di origine prerenale. Si ipotizza inoltre una nefropatia HIV-correlata, peraltro non confermata istologicamente. Gli esami microbiologici dimostrano la presenza della tossina del Clostridium difficile nelle feci. La paziente viene trattata con metronidazolo, con negativizzazione della ricerca della tossina di $C$. difficile. Dopo 4 sedute dialitiche la funzionalità renale e gli esami ematochimici migliorano. Il profilo immunovirologico specifico per l'infezione da HIV mostra un grave deficit immunitario, con linfociti T CD4+ pari a $3 \mathrm{cell} / \mathrm{mm}^{3}$ e HIVRNA pari a 320.000 copie $/ \mathrm{mL}$. Viene pertanto impostata una terapia antiretrovirale con due inibitori nucleosidici della trascrittasi inversa e un inibitore dell'integrasi (tenofovir, emtricitabina e raltegravir). Successivamente risulta positiva anche la viremia del Citomegalovirus (CMV DNA: 18.750 copie $/ \mathrm{mL}$ ) e la paziente sviluppa un'aspergillosi polmonare; viene pertanto posta la diagnosi di AIDS conclamato e si inizia il trattamento con ganciclovir endovena a dosaggio ridotto in base alla clearance renale e voriconazolo al dosaggio usuale.

Al sedicesimo giorno di ricovero si verificano crisi ipertensive di difficile controllo, associate a crisi comiziali subentranti e cecità centrale. La tomografia computerizzata (TC) eseguita in urgenza mostra ipodensità diffusa della sostanza bianca periventricolare, particolarmente evidente in sede occipitale bilaterale ( fig. 1); l'elettroencefalogramma (EEG) evidenzia segni elettrici di sofferenza cerebrale diffusa a massima espressione posteriore, maggiori a sinistra, con

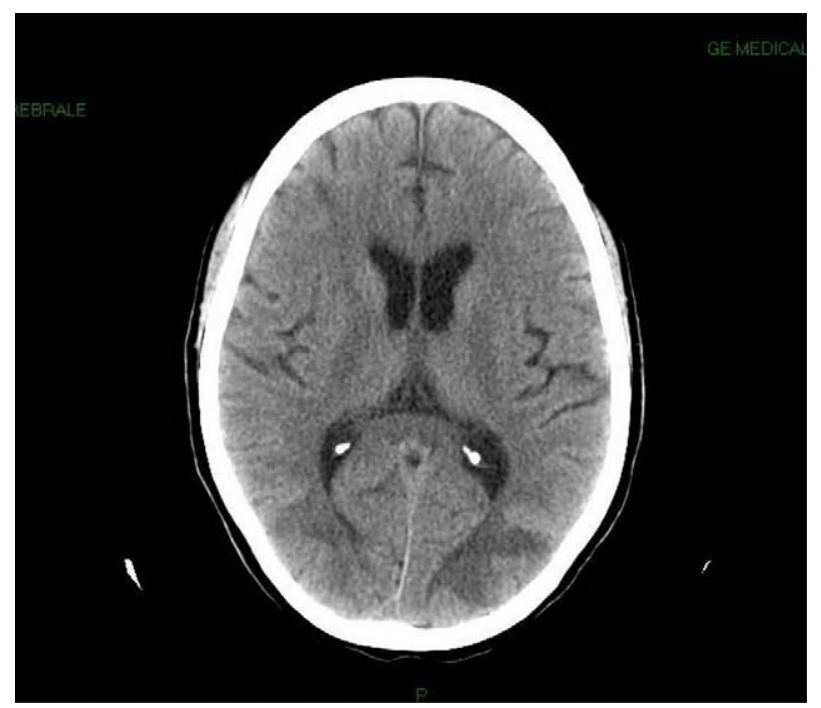

Figura 1 TC senza mezzo di contrasto: ipodensità diffusa della sostanza bianca in sede occipitale bilaterale.

aspetto di tipo epilettiforme; l'esame del liquor mostra assenza di cellularità, glicorrachia $43 \mathrm{mg} / \mathrm{dL}$, proteinorrachia 5,80 mg/dL; negative risultano la ricerca molecolare di virus neurotropi e la liquorcoltura.

La consulenza neurologica, alla luce del referto EEG e della TC, pone il sospetto di una sindrome neurologica inquadrabile come PRES. Infine, la risonanza magnetica nucleare (RMN) mostra la presenza di alterazioni di segnale diffuse, alcune di aspetto digitato, coinvolgenti la sostanza bianca profonda periventricolare, i centri semiovali e la sostanza bianca cortico-sottocorticale emisferica bilateralmente, iperintense nelle sequenze a lungo TR e iso-ipointensa in T1w. Nelle sequenze pesate DWI è presente restrizione della diffusività con aspetto "giriforme" in sede cortico-sottocorticale parieto-occipitale bilaterale, maggiore a destra (fig. 2).

La paziente viene quindi trattata con antipertensivi e anticonvulsivanti; dopo la stabilizzazione della pressione arteriosa, la sindrome neurologica regredisce progressivamente nelle 2 settimane successive.

\section{Discussione}

La PRES è una sindrome neurologica caratterizzata da uno specifico quadro radiologico e descritta per la prima volta nel 1996 [1]. Clinicamente si presenta con un esordio acutosubacuto caratterizzato da cefalea, letargia, sonnolenza o agitazione, amnesia, alterazioni dello stato di coscienza, vomito, convulsioni, coma, alterazioni del sensorio, cecità. Le convulsioni segnano spesso l'esordio della patologia, ma possono comparire anche successivamente, focali o generalizzate. Nell' $80 \%$ dei casi descritti in letteratura la sindrome compare in corrispondenza di crisi ipertensive e, una volta stabilizzata la pressione arteriosa con la terapia farmacologica, la sintomatologia neurologica regredisce e il quadro neuroradiologico si normalizza. Per questo, fra le diverse ipotesi patogenetiche, la più avvalorata sostiene che innalzamenti bruschi della pressione sistemica possano sfuggire al 

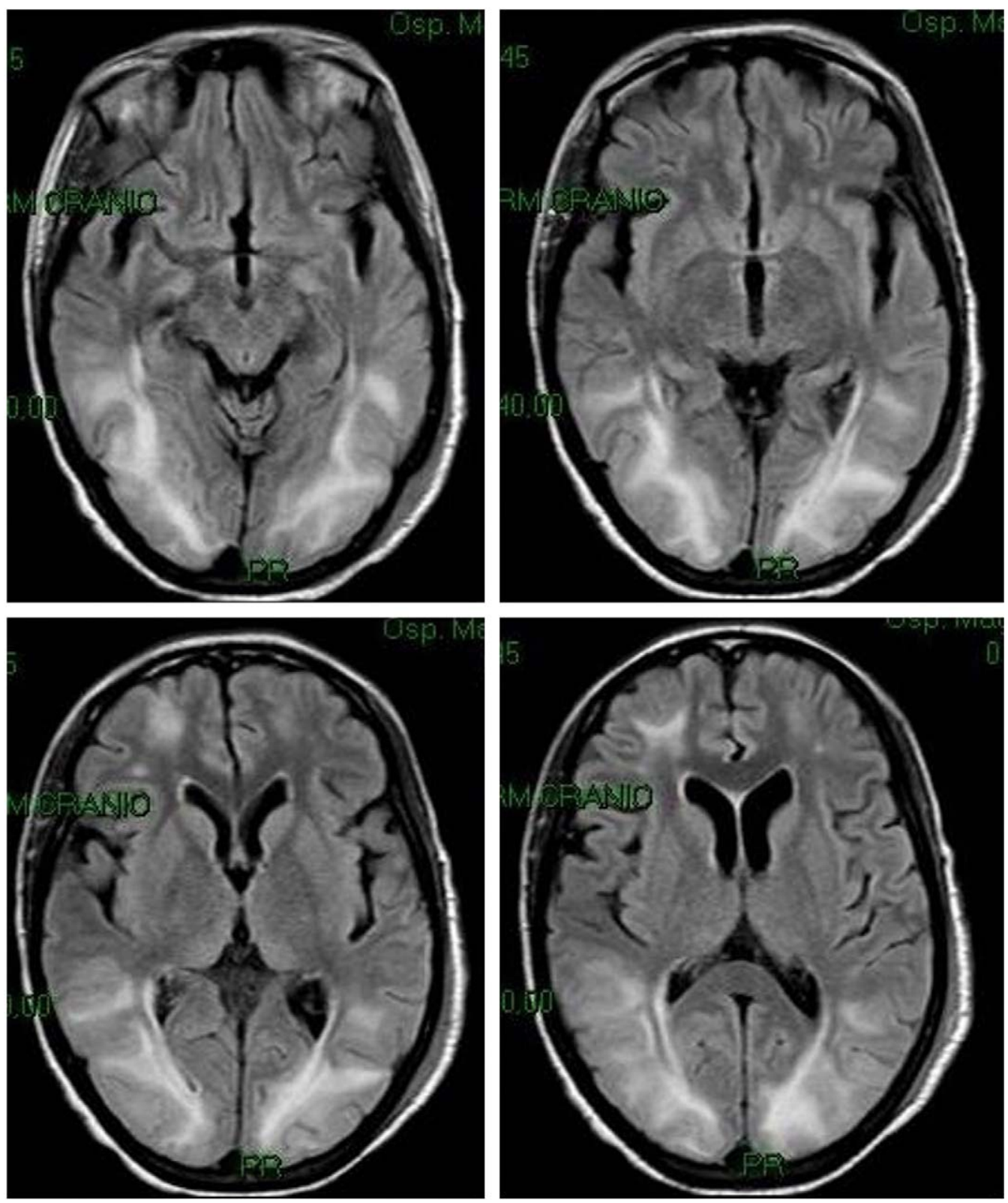

Figura 2 RMN, sequenze assiali FLAIR T2 pesate: alterazioni di segnale diffuse iperintense.

meccanismo di autoregolazione delle arterie cerebrali [2]. L'autoregolazione è una funzione intrinseca dei vasi cerebrali, che garantisce una stabilità del flusso ematico cerebrale in presenza di sbalzi di pressione sistemica. Questa funzione è regolata dalle cellule endoteliali tramite il rilascio di fattori che inducono la vasodilatazione (ossido nitrico) e fattori che determinano la vasocostrizione (trombossano A2 ed endotelina). In presenza di aumenti di pressione arteriosa oltre i limiti di autoregolazione del flusso cerebrale, si ha un'eccessiva dilatazione delle arteriole con danno del letto capillare e formazione di edema vasogenico.

La disfunzione endoteliale è un elemento predisponente comune fra le varie condizioni a rischio di PRES. Infatti, questa sindrome, associata inizialmente all'eclampsia, all'effetto tossico di alcuni immunosoppressori come la ciclosporina e il tacrolimus, e al lupus eritematoso sistemico, è oggi descritta in una varietà di condizioni cliniche tra cui le infezioni croniche, lo shock settico, le malattie autoimmuni (sclerosi sistemica, granulomatosi di Wegener, poliarterite nodosa), la chemioterapia antitumorale, l'ipercalcemia, l'ipomagnesemia, la sindrome da lisi tumorale [2].

In letteratura sono stati descritti 7 casi di PRES in pazienti sieropositivi [3-8], in 3 dei quali era presente una condizione predisponente allo sviluppo di PRES, cioè porpora trombotica trombocitopenica, ipercalcemia e uso di tacrolimus. In 2 casi, poi, i soggetti erano gravemente immunodepressi e, contestualmente, era stata posta diagnosi di AIDS. Infine, in 3 casi i pazienti erano già in terapia antiretrovirale e non avevano altre condizioni associate.

È ormai noto da numerosi studi che l'incidenza di malattie cardiovascolari è maggiore di almeno 3 volte nei soggetti sieropositivi rispetto alla popolazione generale [9], tuttavia è ancora argomento di discussione se le complicanze cardiovascolari siano una conseguenza dell'infezione da HIV stessa o 
dell'uso a lungo termine della terapia antiretrovirale. Quest'ultima altera il profilo metabolico aumentando i livelli di colesterolo totale, trigliceridi, LDL e VLDL [10], ma il solo effetto di innalzamento dei valori dei lipidi non spiega l'aumentato rischio cardiovascolare [11]. Si suppone, infatti, che il virus stesso possa provocare alterazioni dell'endotelio vascolare, com'è suggerito dalle osservazioni dello studio SMART [12,13], in cui i pazienti sottoposti a interruzioni strutturate della terapia antiretrovirale presentavano un rischio paragonabile a quello di coloro che erano sottoposti continuativamente allo stesso trattamento. L'infiammazione cronica indotta dall'infezione da HIV potrebbe essere un fattore implicato nella progressione del danno vascolare e l'endotelio il teatro di tale evento patogenetico che si esprime tramite la maggiore produzione di citochine infiammatorie e molecole di adesione [14].

Il caso clinico da noi descritto riguarda una paziente con un grave deficit immunitario, un'infezione da HIV nota da almeno 7 anni, citomegalovirosi, aspergillosi polmonare, insufficienza renale da grave disidratazione, che in seguito a puntate ipertensive sviluppa una PRES. In un quadro complesso come questo risulta difficile individuare il peso delle varie patologie, nel provocare la sindrome neurologica.

\section{Conclusioni}

Una raccolta sistematica dei casi di PRES in pazienti sieropositivi per HIV, il confronto con i casi in pazienti portatori di altre patologie e studi volti a individuare $i$ meccanismi tramite cui il virus danneggia l'endotelio vascolare potrebbero aiutare a stabilire il ruolo dell'infezione e della terapia antiretrovirale come condizioni predisponenti allo sviluppo della sindrome.

\section{Bibliografia}

[1] Hinchey J, Chaves C, Appignani B, Breen J, Pao L, Wang A, et al. A reversible posterior leukoencephalopathy syndrome. $N$ Engl J Med 1996;334(8):494-500.

[2] Bartynski WS. Posterior reversible encephalopathy syndrome, part 2: controversies surrounding pathophysiology of vasogenic edema. AJNR Am J Neuroradiol 2008;29(6):1043-9.

[3] Courand PY, Christin F, Ben Cheikh A, Baillon JJ, Ber CE, Rimmelé T. Posterior reversible encephalopathy related to tacrolimus in a liver transplanted HIV patient. Gastroenterol Clin Biol 2010;34(1):29-34.

[4] Ridolfo AL, Resta F, Milazzo L, Caramma I, Matacena G, Antinori $\mathrm{S}$, et al. Reversible posterior leukoencephalopathy syndrome in 2 HIV-infected patients receiving antiretroviral therapy. Clin Infect Dis 2008;46(2):e19-22.

[5] Tanioka R, Yamamoto Y, Sakai M, Makie T, Mori M, Uehira T, et al. Convalescence of atypical reversible posterior leukoencephalopathy syndrome in human immunodeficiency virus infection. $J$ Med Invest 2007;54(1-2):191-4.

[6] Bravo Blanco AM, Zarzalejos Andes JM, Suárez Gago M, Melón Pérez C, Esteban Morcillo J. Reversible posterior leukoencephalopathy syndrome in an HIV-positive patient. Rev Clin Esp 2003;203(5):265-6.

[7] Sylvester SL, Diaz Jr LA, Port JD, Sterling TR. Reversible posterior leukoencephalopathy in an HIV-infected patient with thrombotic thrombocytopenic purpura. Scand J Infect Dis 2002;34(9):706-9.

[8] Giner V, Fernández C, Esteban MJ, Galindo MJ, Forner MJ, Guix $\mathrm{J}$, et al. Reversible posterior leukoencephalopathy secondary to indinavir-induced hypertensive crisis: a case report. Am J Hypertens 2002;15(5):465-7.

[9] Triant VA, Lee H, Hadigan C, Grinspoon SK. Increased acute myocardial infarction rates and cardiovascular risk factors among patients with human immunodeficiency virus disease. J Clin Endocrinol Metab 2007;92(7):2506-12.

[10] Stein JH, Klein MA, Bellehumeur JL, McBride PE, Wiebe DA, Otvos JD, et al. Use of human immunodeficiency virus-1 protease inhibitors is associated with atherogenic lipoprotein changes and endothelial dysfunction. Circulation 2001;104(3): 257-62.

[11] Friis-Møller N, Reiss P, Sabin CA, Weber R, Monforte A, et al., DAD Study Group. Class of antiretroviral drugs and the risk of myocardial infarction. N Engl J Med 2007;356(17):1723-35.

[12] El-Sadr WM, Lundgren JD, Neaton JD, Gordin F, Abrams D, Arduino RC, et al., Strategies for Management of Antiretroviral Therapy (SMART) Study Group. CD4+ count-guided interruption of antiretroviral treatment. N Engl J Med 2006;355(22):228396.

[13] Phillips AN, Carr A, Neuhaus J, Visnegarwala F, Prineas R, Burman WJ, et al. Interruption of antiretroviral therapy and risk of cardiovascular disease in persons with HIV-1 infection: exploratory analyses from the SMART trial. Antivir Ther 2008;13(2):177-87.

[14] de Larrañaga GF, Petroni A, Deluchi G, Alonso BS, Benetucci JA. Viral load and disease progression as responsible for endothelial activation and/or injury in human immunodeficiency virus-1infected patients. Blood Coagul Fibrinolysis 2003;14(1): 15-8. 